\title{
Aged blood inhibits hippocampal function through VCAM1 at blood brain barrier
}

\author{
Guang Yang ${ }^{1}$ and guang.yang ${ }^{2}$ \\ ${ }^{1}$ Imperial College London \\ ${ }^{2}$ PREreview
}

September 26, 2018

Aged blood inhibits hippocampal neurogenesis and activates microglia through VCAM1 at the blood-brain barrier

Hanadie Yousef1, Cathrin J Czupalla2, Davis Lee3, Ashley Burke1, Michelle Chen4, Judith Zandstra1, Elisabeth Berber1,3, Benoit Lehallier1, Vidhu Mathur1, Ramesh V Nair5, Liana Bonanno1, Taylor Merkel1, Markus Schwaninger6, Stephen Quake4, Eugene C Butcher2,3, Tony Wyss-Coray $1,3 *$

bioRxiv preprint first posted online Jan. 3, 2018; doi: http://dx.doi.org/10.1101/242198.

Humanity has been seeking fountain of youth through the ages, and nowadays many promising findings against aging have been made public. However, with increasing life expectance, cognitive decline continues to be one of the most concerning health challenges. Aged-related neurodegeneration, typically in hippocampus, is responsible for many geriatric brain diseases such as Alzheimer's disease. The hippocampus subserves learning and memory, and is always the first region of brain to suffer injury (Castellano et al., 2017). Due to its vulnerability to adverse effect of aging, hippocampus has become one of the most important targets of attempts to remit aging damages to the brain. Previous studies have discovered the rejuvenation effects of young blood as it can revitalize hippocampus function in aged mice treated with plasma of young mice (Castellano et al., 2017; Villeda et al., 2011). Conversely, some studies also reveled that besides natural aging, hippocampus deterioration could also be driven by treatments of plasma from aged individuals(Villeda et al., 2011; Rebo et al., 2016) . Early in 2005, an experiment through vascular anastomoses of young and aged mice has proposed the idea that old plasma relies on systemic inhibitory factors to degenerate organs, including the brain, in young mice (Conboy et al., 2005).

A recent research carried out by Yousef et al. (2018) in Stanford University also observed sufficient capacity of aged plasma to trigger aging phenotypes in young brains, primarily in neurogenesis suppression and microglia activation of the hippocampus. Activated microglia is known to be a chronic source of diverse neurotoxins that leads to neuronal function loss, particularly in aged brain and neurodegenerative diseases (Lull and Block, 2010). These two phenomena are considered to be cellular hallmarks of brain aging. Yousef et al. (2018) hypothesized that deterioration of hippocampus function in aged circulatory environment is mediated by blood-brain barrier (BBB). Because BBB separates brain from blood and protects brain parenchyma from harmful factors in the circulating milieu. In their paper, Yousef et al. (2018)explained that factors in blood affect brain cells through behaviour of brain endothelial cells (BECs) constituting the BBB.

To determine the proteins involved in age-related changes in BECs, Yousef et al. (2018) compared the plasma proteomic differences between healthy aging control groups and identified 31 protein factors related significantly with age. Among them soluble form of Vascular Cell Adhesion Molecule 1 (sVCAM1) has 
performed the most strongly positive correlation with age. However, aged plasma depleted of sVCAM1 did not display obviously less detrimental effects on young brain, indicating that sVCAM1 is not the driving factor of aging phenotype. (Yousef et al., 2018) explained the high quantity of sVCAM1 as a result of high membrane bound Vascular Cell Adhesion Molecule 1 (VCAM1) because of the constitutive shedding of VCAM1 from BBB to plasma (Garton et al., 2003; SINGH et al., 2005)(SINGH et al., 2005). This is in line with the higher expression level of VCAM1, according to higher Vcam1 mRNA concentration detected in aged BECs compared to young ones.

(Yousef et al., 2018)discovered that cultured BECs treated with aged plasma performed considerably higher level of VCAM1 than those treated with young plasma. Similar results can be observed in vivo when infusing aged plasma into young mice. VCAM1 is membrane bound on the luminal (blood-facing) side of the BBB and facilitates leukocyte tethering that leads to sustained inflammation of the brain (Yousef et al., 2018). Meanwhile, they also discovered that VCAM1 is upregulated in response to inflammatory effects. Many studies show that aging and aged-related disease are companied by a certain degree of inflammation (Pizza et al., 2011). In nervous system, neuroinflammation refers to the increase of activated microglia while in circulatory system it refers to vascular inflammatory changes. Yousef et al. (2018) suggested that inflamed BECs rely on signally factors transmitting through the BBB to induce subsequent inflammatory response in brain parenchyma. And this signaling is induced by the interaction of VCAM1 and leukocyte. However at this point of their research the signals are not identified.

To determine the significance of VCAM1 in brain degeneration driven by aged plasma, Yousef et al. (2018)deleted Vcam1 gene from young mice and then infused aged or young plasma into these mice. Results of both treatments presented a similar level of neurogenesis and equally infinitesimal quantity of activated microglia. Therefore, Yousef et al. (2018) indicated that Vcam1 deletion is effective to eliminate unfavorable effects on hippocampus caused by aged plasma. Yousef et al. (2018)hypothesized that Vcam1 deletion abrogates negative effects of aged plasma by interfering VCAM1 and leukocyte interaction. To test this hypothesis, they systemically administrated monoclonal VCAM1 antibody into young mice. With VCAM1 antibody treatment, the neurogenesis inhibition and microglia activation triggered by aged plasma treatment were both prevented, while the increase in VCAM1 expression remained unaffected. More excitingly, treatment of VCAM1 antibody also improved neurogenesis and reduced active microglia number in naturally aged mice. These results revealed that antibody blockage of VCAM1 could effectively mitigate adverse impact of aged plasma and could also rejuvenate aged brains. Based on the above findings, Yousef et al. (2018) proposed a model explaining the mechanism of how aged plasma influence brain cell behaviour through VCAM1 at the BBB and how VCAM1 antibody inhibits the influences (Fig.1).

This research is a further proof of previous studies on inhibitory effects of aged blood on young brains. Inspiringly, Yousef et al. (2018)proposed a model of the mechanism that aged plasma trigger aged-related brain damage through a specific protein at the blood-brain barrier. In traditional treatments of neurodegeneration diseases, blood brain barrier remains the biggest obstacle of therapeutic interference to the brain. Importantly, Yousef et al. (2018) revealed the possibility to ameliorated age-related neurogenesis decline and microglia activity via noninvasive modulation of proteins at blood brain barrier. Therefore, their research provided a possible alternative therapy to combat hippocampus neurodegeneration by molecular regulation through circulate system.

The most exciting finding of this paper is that they discovered a particular protein-VCAM1 antibody that reverse age-related brain dysfunction in vivo, which has a promising future to clinical application. From the perspective of medicinal development, this VCAM1 antibody molecule has already been approved in treatments for Multiple Sclerosis (MS) and Crohn's Disease (Polman et al., 2006), supporting the feasibility and safety of this antibody. With multiple previous researches1-4, this is one further step towards human rejuvenation and the 'fountain of youth'. However, many details of the proposed mechanism behind this process remain unclear.

In their proposed model of VCAM1 modulation, signals leading to neurodegeneration are considered to be induced by leukocyte and VCAM1 interactions through VLA-4 integrin (Yousef et al., 2018). However, 
systemic administration of VLA-4 antibody into aged mice showed different results compared to VCAM1 antibody treatment (Yousef et al., 2018). VLA-4 intervention only reduced active microglia and did not affect neurogenesis. This result may indicate that leukocytes bind to VCAM1 through multiple receptors or pathways. Alternatively it might suggest that the signaling is not necessarily triggered by VCAM1 via leukocyte interaction, but possibly depend on other VCAM1 behaviour. Moreover, the inhibitory factors in aged plasma as well as the signal factors through blood brain barrier involved in this model are not yet identified in this paper. As there some factors remain unknown in this mechanism, the proposed model by Yousef et al. (2018) still needed to be verified through further researches.

Yousef et al. (2018)constructed the linkage between VCAM1 and brain crossing the blood brain barrier, which indicates VCAM1 as potential target for age-associated brain disease treatment. This research also provided impressive evidence for medical implication for strategies to alleviate, or even reverse age-associated neurodegeneration. Although there are some questions left in this research, it is still of great importance to future academic studies and clinical application. 


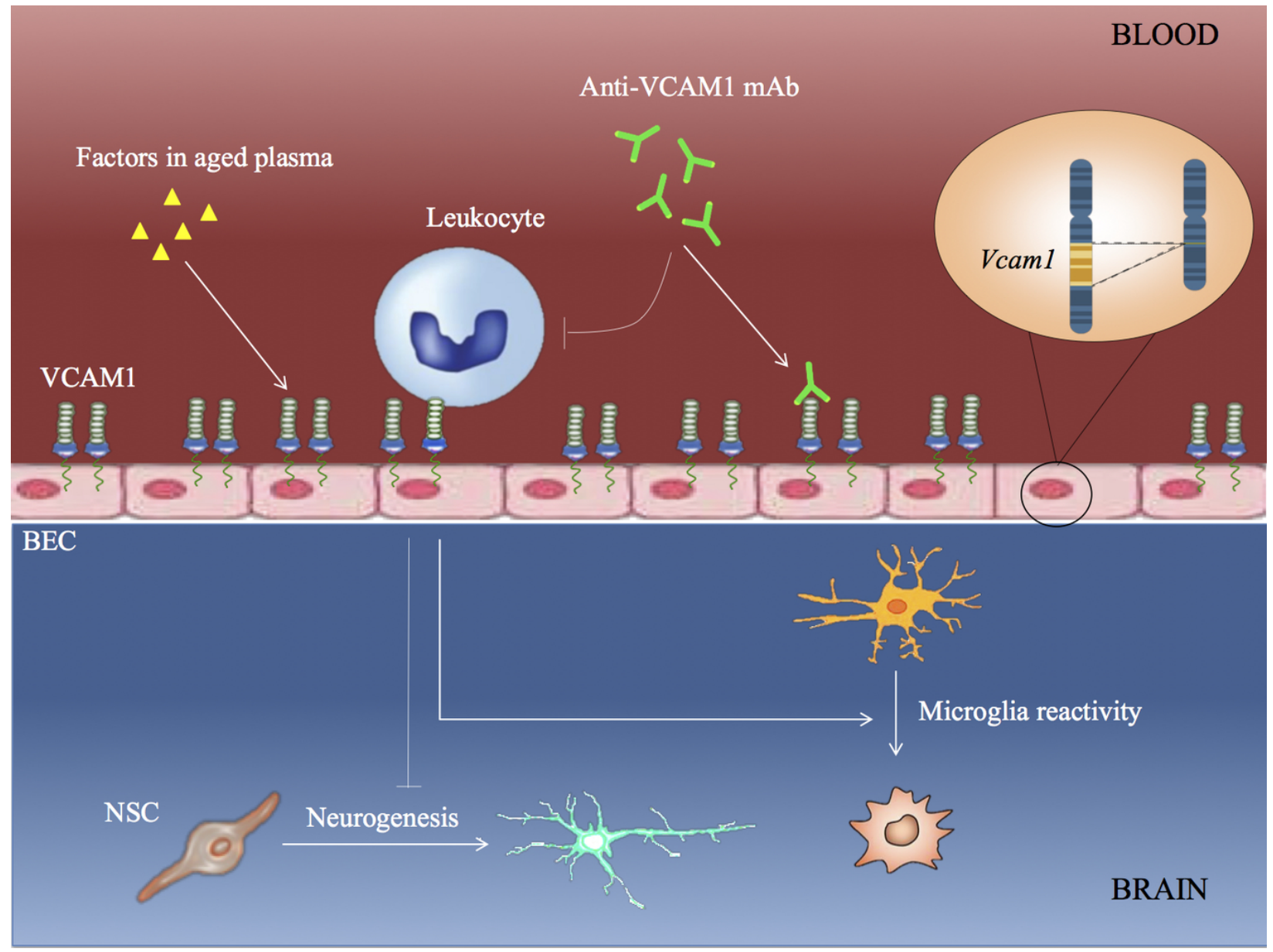

Figure 1: Proposed model of aged plasma inhibits neurogenesis and activates microglia via up-regulation of VCAM1 expression in Brain Endothelial Cells (BECs). Plasma substance on the luminal side of blood brain barrier (BBB) can affect the brain homeostasis on the other side through VCAM1. Factors in aged plasma induce higher expression level of VCAM1 at BBB. VCAM1 facilitates leukocytes tethering and inflammation, which leads to suppression of neurogenesis and chronic activation of microglia. VCAM1 antibodies compete with leukocytes for VCAM1 on BECs, mitigating BEC-mediated inflammation during aging or exposure to aged plasma. Vcam1 deletion has equivalent contributions to the protection of brain from adverse effects of aged plasma. 


\section{References}

Joseph M. Castellano, Kira I. Mosher, Rachelle J. Abbey, Alisha A. McBride, Michelle L. James, Daniela Berdnik, Jadon C. Shen, Bende Zou, Xinmin S. Xie, Martha Tingle, Izumi V. Hinkson, Martin S. Angst, and Tony Wyss-Coray. Human umbilical cord plasma proteins revitalize hippocampal function in aged mice. Nature, 544(7651):488-492, apr 2017. doi: 10.1038/nature22067. URL https://doi.org/10.1038\% 2 Fnature22067.

Irina M. Conboy, Michael J. Conboy, Amy J. Wagers, Eric R. Girma, Irving L. Weissman, and Thomas A. Rando. Rejuvenation of aged progenitor cells by exposure to a young systemic environment. Nature, 433 (7027):760-764, feb 2005. doi: 10.1038/nature03260. URL https://doi.org/10.1038\%2Fnature03260.

Kyle J. Garton, Peter J. Gough, Julie Philalay, Paul T. Wille, Carl P. Blobel, Robert H. Whitehead, Peter J. Dempsey, and Elaine W. Raines. Stimulated Shedding of Vascular Cell Adhesion Molecule 1 (VCAM-1) Is Mediated by Tumor Necrosis Factor--converting Enzyme (ADAM 17). Journal of Biological Chemistry, 278(39):37459-37464, jul 2003. doi: 10.1074/jbc.m305877200. URL https://doi.org/10.1074\%2Fjbc. m305877200.

Melinda E. Lull and Michelle L. Block. Microglial activation and chronic neurodegeneration. Neurotherapeutics, 7(4):354-365, oct 2010. doi: 10.1016/j.nurt.2010.05.014. URL https://doi.org/10.1016\%2Fj. nurt.2010.05.014.

Vincenzo Pizza, Anella Agresta, Cosimo W. D'Acunto, Michela Festa, and Anna Capasso. Neuroinflammation and Ageing: Current Theories and an Overview of the Data. Reviews on Recent Clinical Trials, 6(3):189-203, sep 2011. doi: 10.2174/157488711796575577. URL https://doi.org/10.2174\% 2F157488711796575577.

Chris H. Polman, Paul W. O'Connor, Eva Havrdova, Michael Hutchinson, Ludwig Kappos, David H. Miller, J. Theodore Phillips, Fred D. Lublin, Gavin Giovannoni, Andrzej Wajgt, Martin Toal, Frances Lynn, Michael A. Panzara, and Alfred W. Sandrock. A Randomized Placebo-Controlled Trial of Natalizumab for Relapsing Multiple Sclerosis. New England Journal of Medicine, 354(9):899-910, mar 2006. doi: 10.1056/nejmoa044397. URL https://doi.org/10.1056\%2Fnejmoa044397.

Justin Rebo, Melod Mehdipour, Ranveer Gathwala, Keith Causey, Yan Liu, Michael J. Conboy, and Irina M. Conboy. A single heterochronic blood exchange reveals rapid inhibition of multiple tissues by old blood. Nature Communications, 7:13363, nov 2016. doi: 10.1038/ncomms13363. URL https://doi.org/10. $1038 \% 2$ Fncomms 13363 .

R SINGH, J MASON, E LIDINGTON, D EDWARDS, R NUTTALL, R KHOKHA, V KNAUPER, G MURPHY, and J GAVRILOVIC. Cytokine stimulated vascular cell adhesion molecule-1 (VCAM1) ectodomain release is regulated by TIMP-3. Cardiovascular Research, 67(1):39-49, jul 2005. doi: 10.1016/j.cardiores.2005.02.020. URL https://doi.org/10.1016\%2Fj . cardiores. 2005.02.020.

Saul A. Villeda, Jian Luo, Kira I. Mosher, Bende Zou, Markus Britschgi, Gregor Bieri, Trisha M. Stan, Nina Fainberg, Zhaoqing Ding, Alexander Eggel, Kurt M. Lucin, Eva Czirr, Jeong-Soo Park, Sebastien Couillard-Després, Ludwig Aigner, Ge Li, Elaine R. Peskind, Jeffrey A. Kaye, Joseph F. Quinn, Douglas R. Galasko, Xinmin S. Xie, Thomas A. Rando, and Tony Wyss-Coray. The ageing systemic milieu negatively regulates neurogenesis and cognitive function. Nature, 477(7362):90-94, sep 2011. doi: 10.1038/nature10357. URL https://doi.org/10.1038\%2Fnature10357.

Hanadie Yousef, Cathrin J Czupalla, Davis Lee, Ashley Burke, Michelle Chen, Judith Zandstra, Elisabeth Berber, Benoit Lehallier, Vidhu Mathur, Ramesh V Nair, Liana Bonanno, Taylor Merkel, Markus Schwaninger, Stephen Quake, Eugene C Butcher, and Tony Wyss-Coray. Aged blood inhibits hippocampal neurogenesis and activates microglia through VCAM1 at the blood-brain barrier. jan 2018. doi: 10.1101/242198. URL https://doi.org/10.1101\%2F242198. 